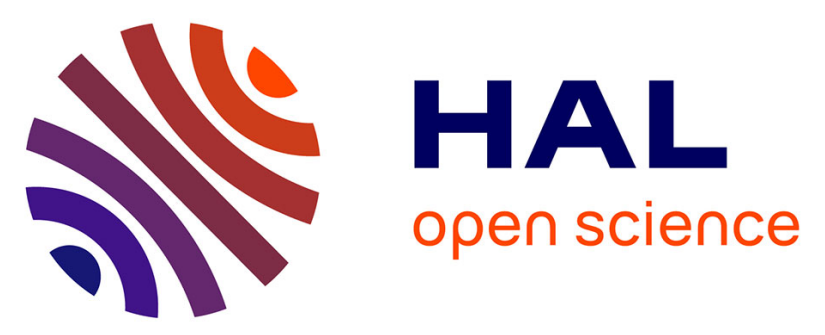

\title{
Two-phase model of liquid-liquid interactions with interface capturing: Application to water assisted injection molding
}

Luisa Silva, Rodolf Lanrivain, Walid Zerguine, Andrès Rodriguez-Villa, Thierry Coupez

\section{To cite this version:}

Luisa Silva, Rodolf Lanrivain, Walid Zerguine, Andrès Rodriguez-Villa, Thierry Coupez. Two-phase model of liquid-liquid interactions with interface capturing: Application to water assisted injection molding. Materials Processing and Design, Modeling, Simulation and Applications, NUMIFORM '07: 9th International Conference on Numerical Methods in Industrial Forming Processes, Jun 2007, Porto, Portugal. pp.Pages 361-368, 10.1063/1.2740838 . hal-00510565

\section{HAL Id: hal-00510565}

https: / hal-mines-paristech.archives-ouvertes.fr/hal-00510565

Submitted on 1 Apr 2011

HAL is a multi-disciplinary open access archive for the deposit and dissemination of scientific research documents, whether they are published or not. The documents may come from teaching and research institutions in France or abroad, or from public or private research centers.
L'archive ouverte pluridisciplinaire HAL, est destinée au dépôt et à la diffusion de documents scientifiques de niveau recherche, publiés ou non, émanant des établissements d'enseignement et de recherche français ou étrangers, des laboratoires publics ou privés. 


\title{
Two-Phase Model of Liquid-Liquid Interactions With Interface Capturing: Application to Water Assisted Injection Molding
}

\author{
Luisa Silva, Rodolphe Lanrivain, Walid Zerguine, Andrès Rodriguez-Villa and \\ Thierry Coupez
}

\begin{abstract}
CEMEF - Ecole des Mines de Paris - UMR CNRS 7635, rue Claude Daunesse, 06904 Sophia-Antipolis, France FEUP, Faculdade de Engenharia, Universidade do Porto, Rua Dr Roberto Frias s/n, 4200-465 Porto, Portugal Transvalor SA, 694 AV. Dr MauriceDonat, 06255 Mougins, France
\end{abstract}

\begin{abstract}
In this paper, a two phase model to compute liquid-liquid flows is presented. We consider that one phase is a highly viscous thermodependent liquid (polymer phase), whereas the second one is a low viscosity low temperature fluid (water). The first part of this paper concerns capture of the interface between the water and the polymer (or determination of the phase field function). Classical VOF and Level set techniques have been implemented and were ameliorated using mesh adaptation techniques. To accurately determine the velocity field, a two-phase formulation is considered, based in the theory of mixtures, and we introduce a scalar parameter, the phase fraction quantifying the presence of each phase in each point of the computational domain. A friction type coupling between both phases is retained. Using the mixed finite element method within an eulerian framework, we calculate in a single system the whole kinematic variables for both liquids (velocity and pressure of each phase). Results are shown, for 2D and 3D parts.
\end{abstract}

Keywords: Water Assisted Injection Molding, Finite element method, Two phase flow.

PACS:

\section{INTRODUCTION}

Water Assisted Injection Molding (WAIM) is a recent injection process, similar to Gas Assisted Injection Molding, but that presents a number of advantages: for example, water allows a rapid cooling and a reduction of cycle times; water incompressibility gives higher pressures and more uniform veins. However, the WAIM technique is not well mastered today, and simulation is an important contribution for its development. One of the main problems in WAIM simulation is the inaccurate determination of the water vein position throughout the process.

In the first part of this paper, a technique to accurately determine the position of the interface in the computational mesh is described. Secondly, to further improve the accuracy in this position, the mesh is adapted and to deal with the difference in the viscosities of both fluids that can lead to numerical problems, a two phase formulation can be used.
Finally, some results in water injection are shown for simple and complex parts.

\section{WATER/POLYMER INTERFACE DETERMINATION}

In an eulerian context, we may consider two main types of interface capturing techniques: a Volume OF Fluid (VOF) method and a Level Set technique.

VOF methods are obtained by considering the distribution of the volume fraction; the Level Set methods are based on a function from which the level zero traces is the interface that we wish to describe. In both cases the advection equation (1) is used to transport either the volume fraction on each element, or the level set zero, with $\mathrm{v}$ the velocity field.

$$
\frac{\partial \alpha}{\partial t}+v_{\text {convection }} \cdot \nabla \alpha=0
$$

In the case of the VOF method, we consider that our computational domain $\Omega$ is divided in several 
subdomains $\Omega$ i. We introduce a presence function associated to each phase, $\phi_{1}$. In our case, $\phi_{1}$ is constant and can be interpreted as the filled volume over the total volume of the element K. Equation (1) represents in this case the transport of a VOF quantity with a convection velocity $v$, and since it is a discontinuous quantity, the Discontinuous Galerkin method is used for its resolution [1]. Even if perfectly conservative, its low order interpolation leads to an important numerical diffusion.

If we consider the interface between two fluids, the Level Set technique consists in the introduction of a continuous function $\alpha_{i}$ where the Level Set 0 represents the interface, and $\alpha_{i}$ is usually referred as "signed distance" function. The advection equation (1) is then used to compute the isovalues of $\alpha$. Classical Galerkin schemes are not very adequate to the resolution of pure convection problems, and stabilization techniques as the Streamline Upwind Petrov Galerkin (SUPG) or Residual Free Bubble (RFB) have been used [2].

The Level Set method allows a more accurate representation of the interface than the VOF method, but the solution of the transport equation does not guarantee the distance function property. This is avoided using a reinitialisation scheme.
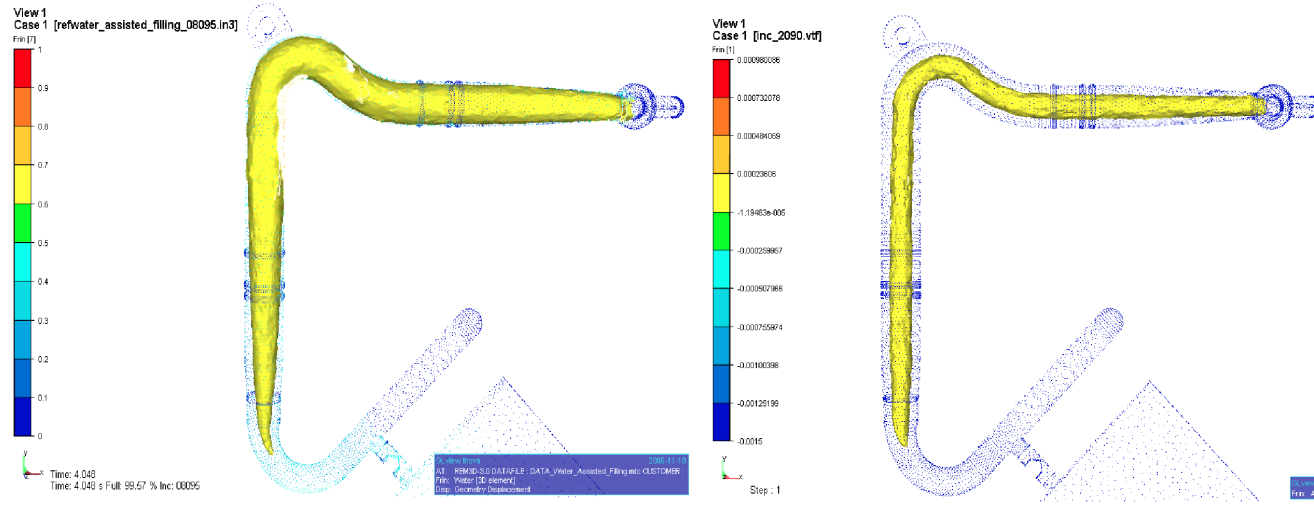

FIGURE 1. Water vein prediction for WAIM of a 3D part. Comparison between a VOF function (on the left) and a Level Set function (on the right), we can observe that there is a tendency to overpredict in the VOF case, due to the inaccuracy when there is high numerical diffusion.
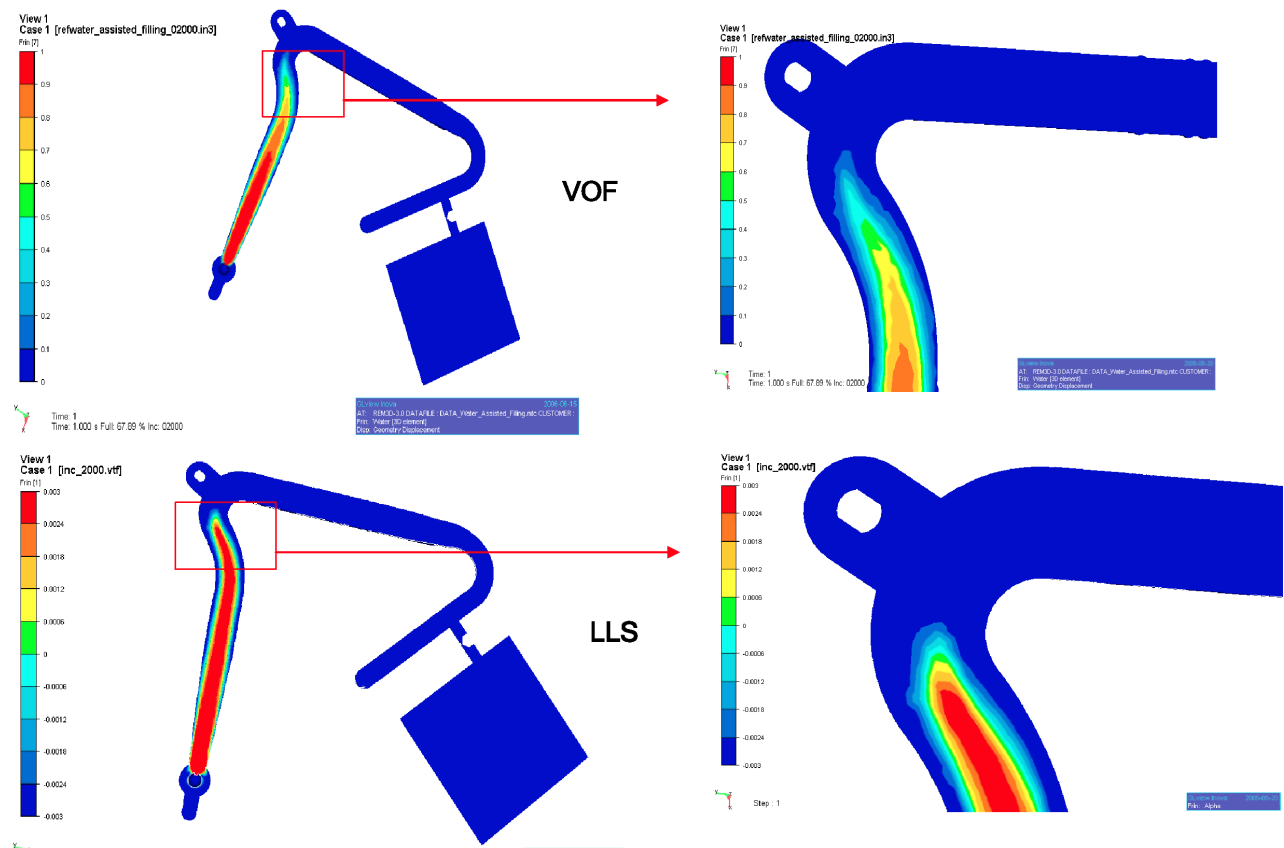

FIGURE 2. In red, we represent water and in blue the polymer. We can notice that the region between both occupies several element layers in the case of the VOF method. 


\section{MESH ADAPTATION TECHNIQUES}

In order to have a good description of the interface water/polymer, two different mesh adaptation techniques can be used: one concerns only optimal nodal displacement, the other anisotropic remeshing. In the first case, the mesh follows the motion of the fluid by contracting the nodes at the interfaces, regaining its original size once the interface passed. Another option is to adapt the mesh anisotropically (hadaptation) using a metric field of the form: $M=m^{2} A+\varepsilon^{2} I$, where $A=\nabla \alpha \otimes \nabla \alpha$, and $\varepsilon$ the default size [4]. We can control the number of element layers through parameters $m$ and $\varepsilon$. Figure 3 illustrates the mesh obtained using this kind of approach, in a Water Assisted Injection molding example.
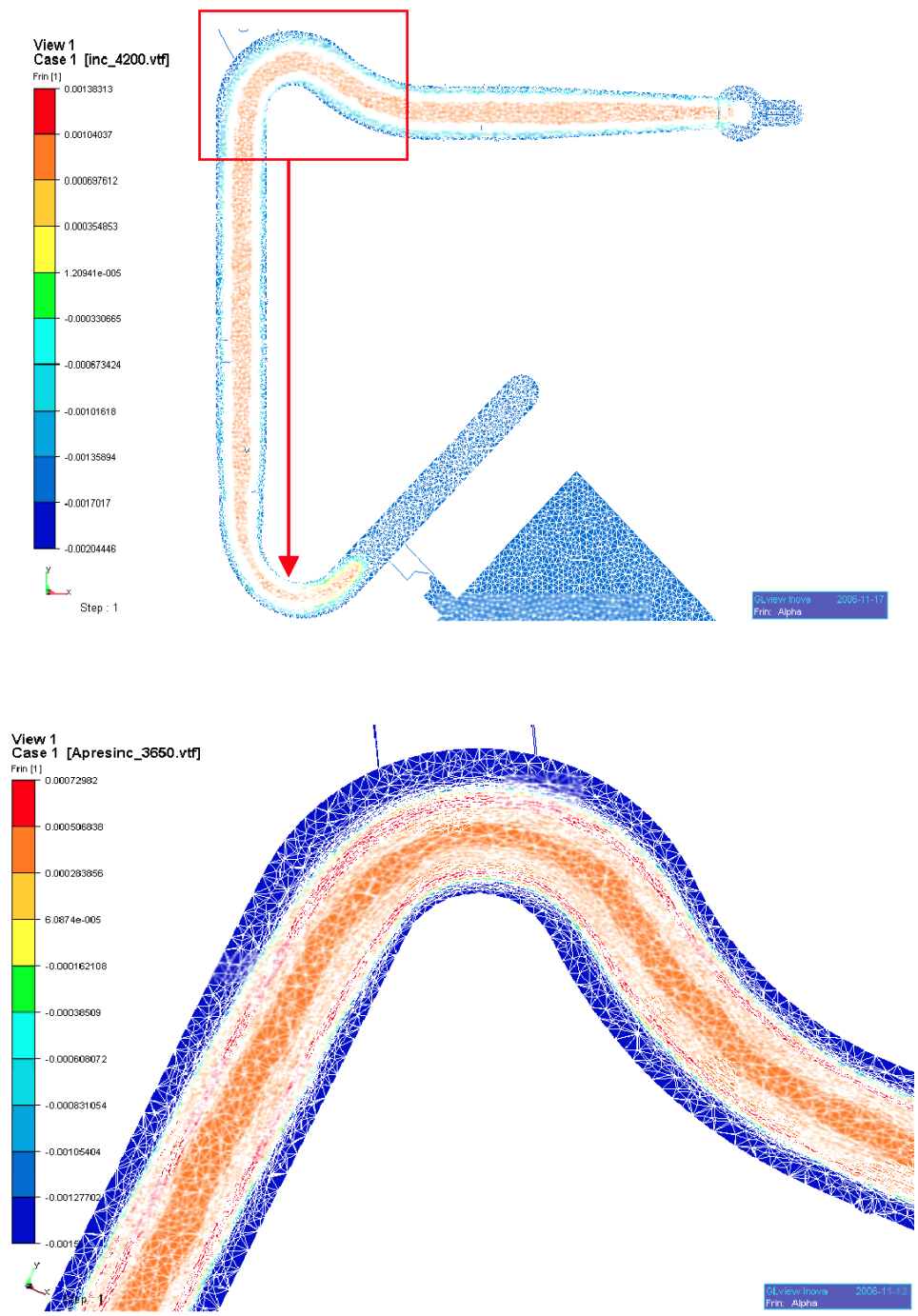

FIGURE 3. Representation of the mesh in a transversal cutting plane of a 3D WAIM part. Below, a detail showing the number of anisotropic layers. 


\section{TWO-PHASE FORMULATION}

Let us consider a $\Omega$ domain constituted of two liquid phases, one that has a low viscosity, one with a higher one, respectively $\Omega_{1}$ and $\Omega_{2}$. Supposing the saturated medium hypothesis, only these two phases are present in each point of the domain. According to the theory of mixtures framework, we introduce the volume fraction of the phase $i$, already referred above, and defined as

$$
\phi_{i}(x, t)= \begin{cases}1 & \text { if } \quad x \in \Omega_{i} \\ 0 & \text { else }\end{cases}
$$

Neglecting the inertia and compressibility terms for a question of simplification and splitting the stress into two liquids contribution, the dynamic balance of the medium is equivalent to a system of two equilibrium equations, one for each phase:

$$
\left.\begin{array}{l}
\nabla \cdot \sigma=0 \\
\sigma=\sigma_{1}+\sigma_{2}
\end{array}\right\} \Rightarrow \nabla \cdot \sigma_{1}=-\nabla \cdot \sigma_{2}=F
$$

or

$$
\left\{\begin{array}{l}
\nabla \cdot \sigma_{1}=F \\
\nabla \cdot \sigma_{2}=-F
\end{array}\right.
$$

Each liquid is thus described by the system

$$
\left\{\begin{array}{l}
\nabla \cdot\left[2 \eta_{i} \varepsilon\left(v_{i}\right)\right]-\nabla p_{i}=F \\
-\nabla \cdot v_{i}=0
\end{array}\right.
$$

We choose to model $F$ as a friction force. This force describes the action-reaction between the two liquids and is given by:

$$
F=k\left(v_{1}-v_{2}\right)
$$

where $k \mathrm{~s}$ an interaction coefficient between the two phases. This force acts in the liquid-liquid interface (even if the interface is diffuse). We can transform the surface integration into a volume integration by multiplying by $\phi(1-\phi)$.
The system describing the whole kinematics variables of the problem is obtained by re-writing system (5) on each phase and by defining the evolution equation for $\phi$. We obtain then:

$$
\left\{\begin{array}{l}
\phi \nabla \cdot\left[2 \eta_{1} \varepsilon\left(v_{1}\right)\right]-\nabla p_{1}=k\left(v_{1}-v_{2}\right) \\
-\nabla \cdot v_{1}=0 \\
(1-\phi) \nabla \cdot\left[2 \eta_{2} \varepsilon\left(v_{2}\right)\right]-\nabla p_{2}=-k\left(v_{1}-v_{2}\right) \\
-\nabla \cdot v_{2}=0 \\
\frac{d \phi}{d t}=f(x, t)
\end{array}\right.
$$

In this system, variable fields are defined in the whole computational domain. The coupling relation is also extended to the whole domain and makes it possible to ensure the prolongation of the variables on the subdomains (where $\phi=0$ and $\phi=1$ ). The resolution of the evolution equation is decoupled from the conservation equations. Knowing $\phi$ in each point of the domain, we can compute through a single system of equations the four kinematic unknown fields of the problem, using a mixed finite element method.

\section{RESULTS}

Let us consider the water vein development during the injection molding of the test part shown previously. We have launched one step of water vein computation on a coarse mesh, followed by one step of remeshing, and we restart the water computation on the refined mesh. This strategy implies two computations, but avoids remeshing at each time step. Figure 4 shows the difference in the interface description between the second computation and the first one. We observe that the shape of the vein is much smoother and better approximated than the one obtained in the first computation. 

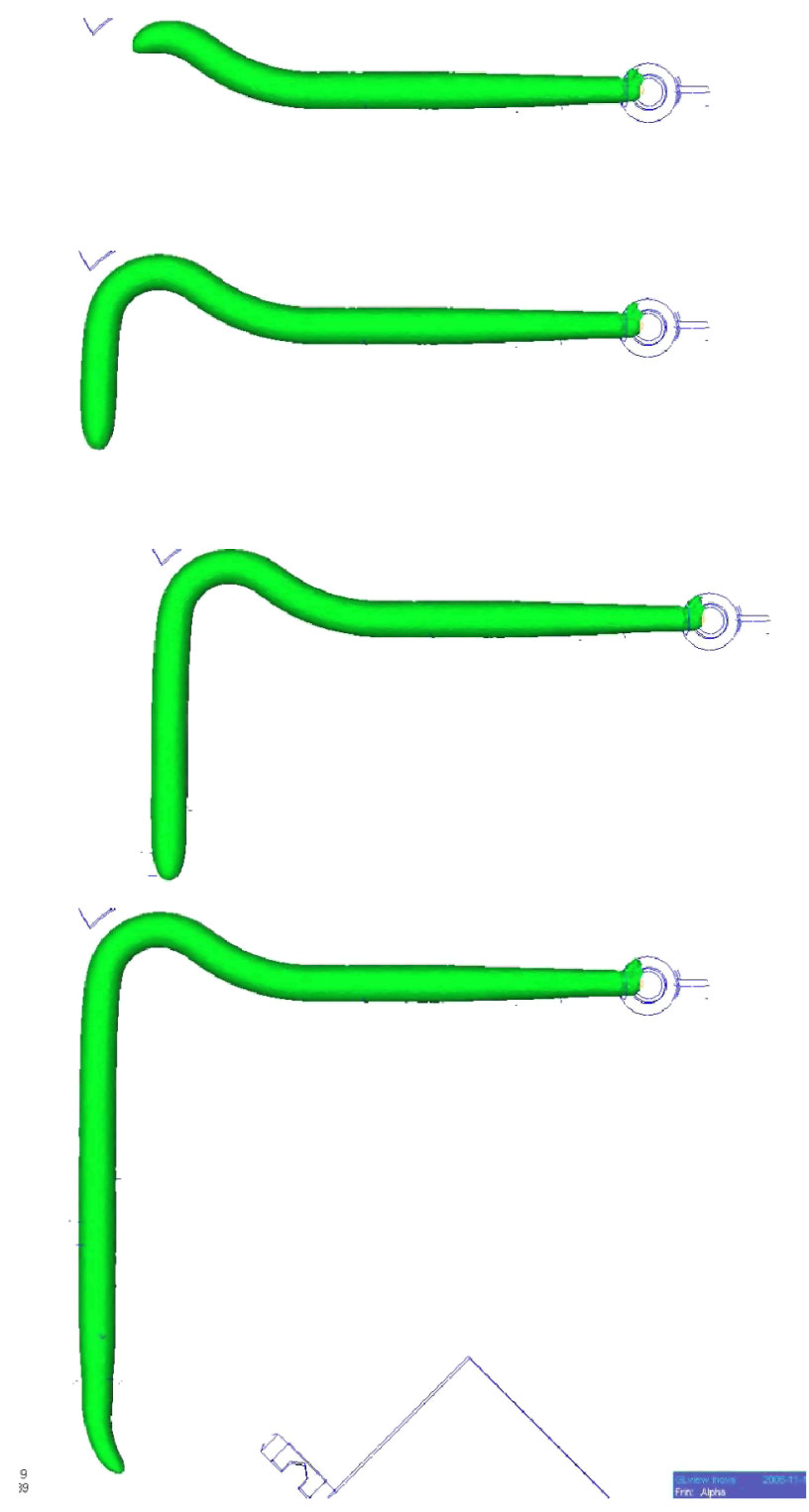

FIGURE 4. Water development after and before remeshing.
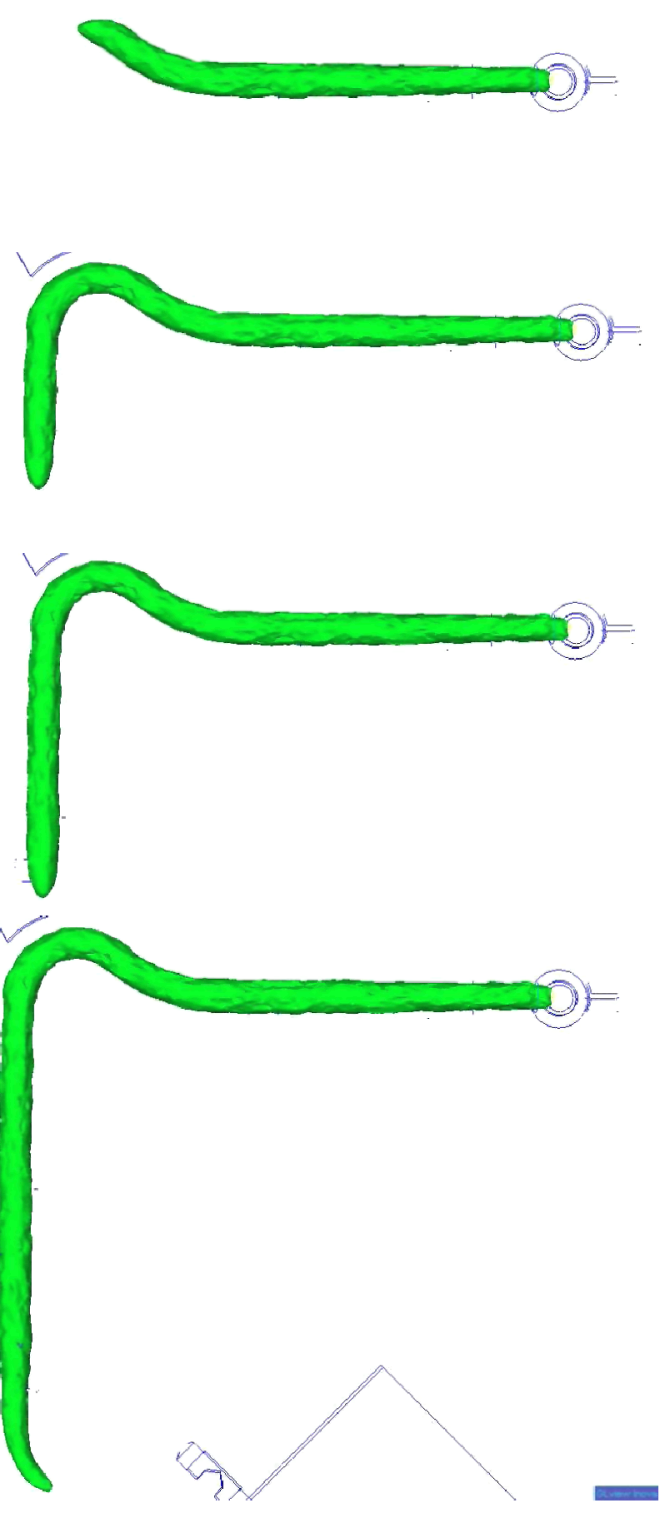


\section{CONCLUSIONS}

We have used a h-adaptation technique, that combined with a level set function and a two phase formulation allows an accurate determination of the water vein in the WAIM processes. Next developments include taking into account the solidified layer of polymer by extending the two phase formulation to the solid case.

\section{ACKNOWLEDGMENTS}

The authors would like to acknowledge the CETIM and TRANSVALOR for the financial support of this work.

\section{REFERENCES}

1. S. Batkam, J. Bruchon and T. Coupez, Int. J. Form. Processes 7(1-2), pp. 11 (2003).

2. O. Basset, H. Digonnet and T. Coupez, Coupled Problems 2005 Proceedings, Santorini 2005.

3. E. Bigot and T. Coupez, Eccomas 2000, Barcelona 2000.

4. C. Gruau and T. Coupez, Comp. Meth. Appl. Mech. Eng., 194(48-49), pp.4951 (2005) 